\title{
A systematic review of childhood and adolescent cohorts: a comparison of reported energy and macronutrient intakes
}

\author{
C.E. Rycroft, C.E.L. Evans and J.E. Cade \\ Nutritional Epidemiology Group, School of Food Science and Nutrition, University of Leeds, LS2 9JT.
}

An estimated $23 \%$ of children in developed countries and $13 \%$ in the developing world are overweight or obese ${ }^{(1)}$ which may adversely affect their health, development and future well-being. This systematic review, registered with PROSPERO: CRD42015030081, sought longitudinal evidence about childhood diet and future obesity risk. As total energy intake is a factor in energy balance, a key inclusion criteria was that childhood and adolescent cohorts measured and quantified the whole diet and could adjust for energy intake in their analyses.

Medline, Embase, Cochrane CENTRAL, Web of Science and Scopus were searched for relevant articles published from 1990. Included full-texts in English were from observational cohort studies of healthy school-aged children which measured whole diet at baseline using 3 day diet records (3dDR), 24 hour dietary recalls (24HR) or semi-quantitative food frequency questionnaires (FFQ), measured adiposity at $\geqslant 2$ year follow-up and reported associations between dietary exposure and adiposity outcome. Data was extracted to Excel. Mean energy and macronutrient intakes were compared between cohorts and against UK estimated average requirements $(\mathrm{EAR})^{(2)}$ and $\mathrm{WHO} / \mathrm{FAO}$ population nutrient intake goals ${ }^{(3)}$.

Searches found 11,604 records. After screening 390 full-texts, 35 papers from 14 cohorts (established from 1973 to 2006) were included. 12 cohorts reported baseline energy intakes. Energy intake increased with increasing age of cohort (See figure). Reported baseline mean energy intakes were similar ( $\pm 200 \mathrm{kcal} /$ day) to EAR in pre-teens but less than EAR in mid-teen cohorts. 3 European and 5 USA cohorts also reported macronutrient intakes at baseline. Mean fat intakes (31 to $36 \%$ of total energy) exceeded intake goals (15 to $30 \%$ ). Mean carbohydrate intakes (47 to $54 \%$ of total energy) were lower than intake goals ( 55 to $75 \%$ ).

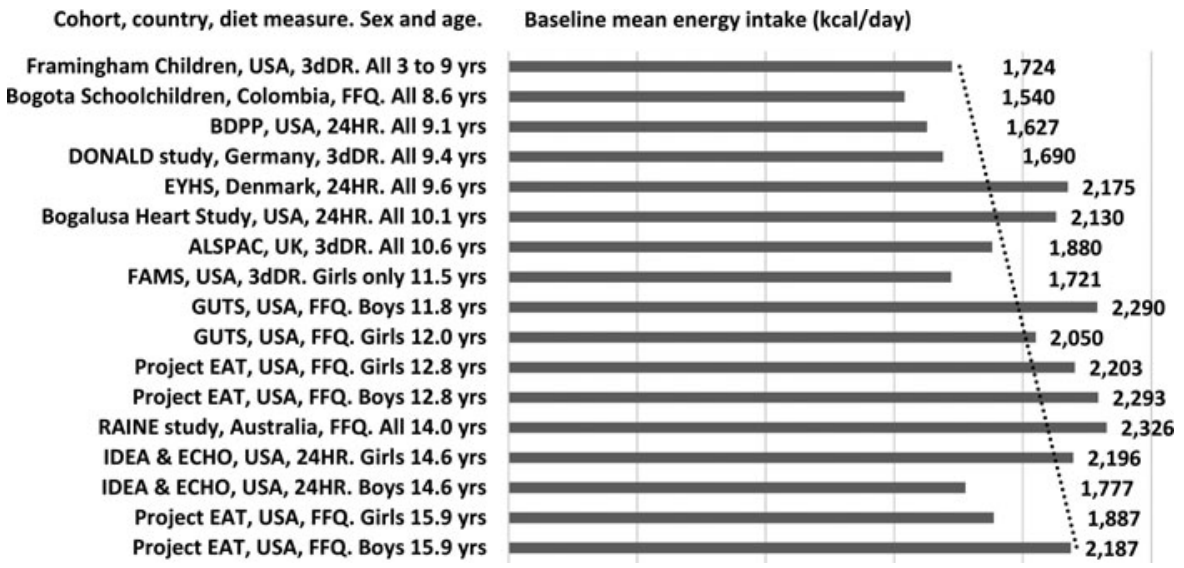

Included cohorts which measured whole diet and later adiposity presented plausible energy intakes, but with signs of underreporting by older children. Most cohorts were from USA and other developed countries and many children had fat intakes above WHO/FAO intake goals. Updated evidence is needed, including good quality evidence from low income countries where prevalence of obesity is increasing more rapidly.

1. Ng M, Fleming T, Robinson M et al. (2014) Lancet 384 (9945), 766-81.

2. SACN (2011) Dietary Reference values for Energy: https://www.gov.uk/government/publications/sacn-dietary-reference-values-for-energy (accessed April 2018).

3. WHO/FAO (2003) Diet Nutrition and the Prevention of Chronic Diseases: http://www.who.int/nutrition/publications/obesity/WHO_TRS_916/en/ (accessed April 2018). 